\title{
Adipose stem cell-derived exosomes exert a synergic anti-inflammatory effect with glucocorticoids and override their detrimental effects on rotator cuff tendons
}

Jinzhong Zhao ( $\sim$ jzzhao@sjtu.edu.cn )

Shanghai Jiao Tong University Affiliated Sixth People's Hospital

Xuancheng Zhang

Shanghai Jiao Tong University Affiliated Sixth People's Hospital

Ang Li

Shanghai Jiao Tong University Affiliated Sixth People's Hospital

Kang Han

Shanghai Jiao Tong University Affiliated Sixth People's Hospital

He Zhang

Fengfeng General Hospital of North China Medical and Health Group

Xiaoqiao Huangfu

Shanghai Jiao Tong University Affiliated Sixth People's Hospital

Jinghuan Huang

Shanghai Jiao Tong University Affiliated Sixth People's Hospital

Jia Jiang

Shanghai Jiao Tong University Affiliated Sixth People's Hospital

\section{Research Article}

Keywords: glucocorticoids, exosomes, rotator cuff tendons

Posted Date: January 28th, 2022

DOI: https://doi.org/10.21203/rs.3.rs-1094362/v2

License: (9) This work is licensed under a Creative Commons Attribution 4.0 International License. Read Full License 


\section{Abstract}

Background: Glucocorticoids (GCs) injections are commonly used to relieve pain and improve function in patients with multiple shoulder disability, they cause detrimental effects on the rotator cuff tendons. Adipose stem cell-derived exosomes (ASC-Exos) reportedly recover impaired tendon matrix metabolism by maintaining tissue homeostasis. It is unclear whether additional ASC-Exos treatment overrides the detrimental effects of GCs without interfering with their anti-inflammatory effects.

Methods: The in vitro studies included inflammatory analysis and cytoprotective analysis. In the inflammatory analysis, rat raw cells were treated with saline, GCs, or GCs + ASC-Exos and evaluated regarding cellular proliferation, migration, and secretion of inflammatory-related cytokines. In the cytoprotective analysis, rat tenocytes were treated with saline, GCs, or GCs + ASC-Exos and evaluated regarding cellular proliferation, migration, senescence, apoptosis, and transcription of tenocytic genes. In the in vivo studies, a subacromial injection of saline, GCs, or GCs + ASC-Exos was performed in a chronic injured-intact rotator cuff rat model. Histological and biomechanical analysis were performed 1 week to evaluate the protective effect of ASC-Exos against GCs-induced detriments on injured-intact in rotator cuffs.

Results: In the in vitro inflammatory analysis, GCs treatment significantly decreased the proliferation, migration, and secretion of pro-inflammatory cytokines in raw cells, and increased the secretion of antiinflammatory cytokines; additional ASC-Exos treatment further significantly decreased the secretion of pro-inflammatory cytokines and increased the secretion of anti-inflammatory cytokines, while restoring GCs-suppressed cellular proliferation and migration. In the in vitro cytoprotective analysis, GCs treatment significantly decreased the proliferation, migration, and transcription of tenocytic matrix molecules of tenocytes, and significantly increased their senescence, apoptosis, and transcription of ROS and tenocytic degradative enzymes; additional ASC-Exos treatment significantly improved the GCs-suppressed cellular proliferation, migration, and transcription of tenocytic matrix molecules, transcription of tenocytic degradative enzyme inhibitors, and significantly decreased the GCs-induced cell senescence, apoptosis, and transcription of ROS and tenocytic degradative enzymes. In the in vivo studies, an additional ASCExos injection restored the impaired histological and biomechanical properties owing to GCs administration.

Conclusion: ASC-Exos may exert a stronger anti-inflammatory effect in combination with GCs, and override their detrimental effects on the rotator cuff.

\section{Background}

Shoulder disability embodies multiple pathologic conditions, including rotator cuff tear, frozen shoulder, calcified tendinitis, and other tendinopathies[1, 2]. Irrespective of the cause, shoulder disability leads to shoulder pain and limited range of motion. The mainstay of treatment is conservative therapy, including rest, activity modification, physical therapy, and local injection of nonsteroidal anti-inflammatory drugs or 
corticosteroids[2-7]. The most common of these conservative therapies is glucocorticoids (GCs) injection[3-5].

Single or repeated GCs injections reportedly significantly reduce shoulder pain and improve shoulder range of motion in athletes with chronic pain[8]. The underlying mechanism is due to the antiinflammatory effect of GCs $[9,10]$. Shoulder injury is followed by a massive infiltration of inflammatory cells and the secretion of several upstream inflammatory cytokines such as TNF-a and IL-1, which triggers a pro-inflammatory reaction and ultimately induces the formation of pain-related neuropeptides[11-14]. GCs achieve their therapeutic effect by inhibiting the infiltration of inflammatory cells and the secretion of upstream pro-inflammatory factors $[9,10,14]$.

Although GCs have potent anti-inflammatory and analgesic effects, they also have important adverse effects. There is increasing concern that GCs injections may accelerate tendon degeneration[2, 6, 15]. Tendon rupture after GCs injections in both the upper and lower extremities is common in clinical practice $[16,17]$. Although it is unclear to what extent such ruptures are attributable to GCs injections or the progression of tendinopathy itself, several studies have confirmed that GC injections are correlated with tendon detriments $[2,6,15]$. Haraldsson et al. showed a significant decrease in the tensile strength of isolated rat-tail collagen fascicles at 3 and 7 days after GCs treatment compared with the saline-treated group[6]. Maman et al. and Mikolyzk et al. reported the detrimental effect of single and repeated GCs injections on both injured and intact rotator cuff tendons[2,15]. The adverse effects of GCs injections on the tendon are believed to be due to the negative effect of GCs on tendon metabolism homeostasis in combination with decreased cell proliferation and increased cell senescence, apoptosis, and destruction of normal collagen organization[2, 6, 7, 15, 18]. Therefore, concomitant treatment with a bioactive substance that overrides the detrimental effects of GCs on cellular metabolism homeostasis may be a practical strategy to minimize the adverse effects of GCs. However, despite considerable research, there is still no efficacious means with which to eliminate the undesirable effects of GCs while retaining their antiinflammatory effects.

Exosomes are nano-sized membrane vesicles that contain proteins, lipids, and nucleic acid and play an important role in the regulation of cellular physiological activities[19-24]. They have attracted increasing interest and have been widely studied in tissue-engineering fields in recent years[19-24]. In our previous studies, we have found that adipose stem cell-derived exosomes (ASC-Exos) were able to recover the impaired matrix metabolism of torn rotator cuff tendons by maintaining tissue homeostasis. Drawing on this, we infer additional treatment with ASC-Exos may override the detrimental effects of GCs on tenocytes without interfering with their anti-inflammatory effects.

The present study aimed to: 1) investigate the anti-inflammatory and cytoprotective effects of ASC-Exos on raw cells and tenocytes, respectively, with the concomitant treatment of GCs in vitro, and 2) evaluate the effect of ASC-Exos on injured-intact tendons with concomitant GCs injection in vivo using a rat model of chronic rotator cuff tear. Our hypothesis was that the additional ASC-Exos treatment would exert a synergic anti-inflammatory effect with the GCs and a cytoprotective effect against their adverse effects 
and would improve both the histological and biomechanical properties of injured-intact rotator cuffs simultaneously injected with GCs in vivo.

\section{Methods}

\section{Isolation and characterization of human ASC-Exos}

ASCs isolation and culture, and ASC-Exo isolation were performed as described in previous studies[25, 26]. The morphology of ASC-Exos was observed using transmission electron microscopy (TEM; $\mathrm{H}-7650$; Hitachi High-Technologies Corporation). The particle size distribution was evaluated using nanoparticle tracking analysis (NTA; ZetaView PMX 120, Particle Metrix, Meerbusch, Germany). The expression of exosomal surface markers, including CD9, CD63, and TSG-101, was detected using western-blotting.

\section{In vitro studies}

\section{Inflammation analysis}

\section{Interventions and study groups}

Raw cells from one of the rat macrophage cell lines were used for inflammation analysis in the present study. After incubation in complete Dulbecco's Modified Eagle's Medium supplemented with $10 \%$ fetal bovine serum (FBS) and antibiotic solution for 24 hours to allow attachment, cells were treated with either saline (control group), $1 \mu \mathrm{M}$ of dexamethasone (GCs group), or $1 \mu \mathrm{M}$ of dexamethasone $+10^{11}$ pellets $/ \mathrm{ml}$ of ASC-Exos (GCs + ASC-Exos group), and further evaluated.

\section{Cell proliferation}

After being seeded in 96-well plates at a density of $1 \times 10^{3}$ cells/well, cells were incubated for 24 hours to allow attachment, and treated with either saline, dexamethasone, or dexamethasone + ASC-Exos. Cell proliferation was evaluated at 12, 24, and 48 hours using a CCK8 assay kit in accordance with the manufacturer's instructions (Dojindo Molecular Technologies, Inc., Kumamoto, Japan). Briefly, at 12, 24, and 48 hours, CCK8 solution was added to each well and incubated for a further 1 hour at $37^{\circ} \mathrm{C}$. The absorbance of wells was measured at $450 \mathrm{~nm}$ using a spectrophotometric microplate reader (Bio-Rad 680 , Bio-Rad, Hercules, CA, USA). The proliferation intensity of the cells was calculated as the optical density of the tested well minus the absorbance of the blank well.

\section{Cell migration}


After being seeded in the upper chamber of 96-well transwell plates at a density of $1 \times 10^{6}$ cells/well, cells were incubated for 24 hours to allow attachment. After this, saline, dexamethasone, or dexamethasone + ASC-Exos was added to the lower chamber. After incubation for another 24 hours, cell migration was evaluated in accordance with the manufacturer's instructions (Corning, Corning, NY, USA). Briefly, cells from the upper surface of the filter membranes were removed with a cotton swab. Cells that migrated to the lower surface of the filter membrane were fixed in $4 \%$ formaldehyde for 5 minutes, and then stained with $0.5 \%$ crystal violet for 5 minutes. Migration activity was evaluated by observing the stained cells under an optical microscope.

\section{Enzyme linked immunosorbent assay (ELISA)}

After being seeded in 96-well plates at a density of $1 \times 10^{5}$ cells/well, cells were incubated for 24 hours to allow attachment and treated with saline, dexamethasone, and dexamethasone + ASC-Exos, respectively. The levels of secreted inflammation-related cytokines, including TNF-a, IL-1a, IL-1 $1 \beta$, IL-4, and IL-10, were evaluated using an enzyme-linked immunosorbent assay kit in accordance with the manufacturer's instructions (R\&D Systems). Briefly, after incubation for another 96 hours, the culture medium was centrifugated to obtain the supernatant. The supernatant was then incubated with specific antibodies for 2 hours at $37^{\circ} \mathrm{C}$ in a 96 -well plate. After washing, each well was incubated with the corresponding conjugate for 1 hour, and substrate solution was added to develop the color in the dark. The absorbance at $450 \mathrm{~nm}$ was determined using a microplate reader (Multiskan MK3; ThermoFisher Scientific, USA).

\section{Cytoprotective analysis}

\section{Interventions and study groups}

Rat tenocytes isolated from adult rats were used in the present study (Appendix). After incubation in complete Dulbecco's Modified Eagle's Medium supplemented with 10\% fetal bovine serum (FBS) and antibiotic solution for 24 hours to allow attachment, cells were treated with either saline (control group), $1 \mu \mathrm{M}$ of dexamethasone (GCs group), or $1 \mu \mathrm{M}$ of dexamethasone $+10^{11}$ pellets $/ \mathrm{ml}$ of ASC-Exos (GCs + ASC-Exos group), and further evaluated.

\section{Cell proliferation}

After being seeded in 96-well plates at a density of $1 \times 10^{3}$ cells/well, cells were incubated for 24 hours to allow attachment and then treated with either saline, dexamethasone, or dexamethasone + ASC-Exos. Cell proliferation was evaluated at 12, 24, and 72 hours using a CCK8 assay kit as described above. 


\section{Cell migration}

After being seeded in the upper chamber of 96-well transwell plates at a density of $1 \times 10^{4}$ cells/well, cells were incubated for 24 hours to allow attachment. Saline, dexamethasone, or dexamethasone + ASC-Exos was then added to the lower chamber. Cell migration was evaluated as described above.

\section{Cell senescence}

After being seeded in 6-well plates at a density of $1 \times 10^{4}$ cells/well, cells were incubated for 24 hours to allow attachment and then treated with saline, dexamethasone, or dexamethasone + ASC-Exos. The senescence assay was performed using a senescence $\beta$-galactosidase staining kit in accordance with the manufacturer's instructions (Solarbio Science and Technology, Beijing, China). Briefly, after incubation for another 24 hours, $1 \mathrm{ml} /$ well of senescence $\beta$-galactosidase staining solution was added to each well and incubated for 15 minutes at $37^{\circ} \mathrm{C}$. Senescent cells were stained with prepared dye and fixed at $37^{\circ} \mathrm{C}$ overnight. Cell senescence was evaluated by calculating the intensity of the positively stained cells under an optical microscope using ImageJ software (National Institutes of Health).

\section{Cell apoptosis}

After being seeded in 96-well plates at a density of $1 \times 10^{4}$ cells/well, cells were incubated for 24 hours to allow attachment before being treated with saline, dexamethasone, or dexamethasone + ASC-Exos. The apoptotic assay was performed using a TUNEL apoptosis assay kit in accordance with the manufacturer's instructions (Solarbio Science and Technology, Beijing, China). Briefly, after incubation for another 24 hours, $50 \mu \mathrm{L} /$ well of TUNEL solution was added to each well and incubated for a further 1 hour at $37^{\circ} \mathrm{C}$. Apoptotic cells were stained with reaction buffer, and the fluorescence intensity was evaluated using a flow cytometer with a FL3 channel.

\section{Real-time polymerase chain reaction (Rt-PCR)}

After being seeded in 6-well plates at a density of $1 \times 10^{6}$ cells/well, cells were incubated for 24 hours to allow attachment. Cells were then treated with saline, dexamethasone, or dexamethasone + ASC-Exos and incubated for a further 72 hours. The relative mRNA transcription levels of ROS, MMP-2, MMP-9, MMP-13, TIMP-1, TIMP-3, decorin, biglycan, and type I and type III collagen were evaluated as previously described[26]. Briefly, TRIzol reagent (Invitrogen, Carlsbad, CA, USA) was used to extract the total RNA from cells. Reverse transcription was performed using script reverse transcription supermix (Bio-Rad Laboratories, Hercules, CA, USA) to reverse-transcribe the RNA into cDNA. Real-time polymerase chain reaction was conducted using the CFX Connect Real-Time PCR system (Bio-Rad) with Universal SYBR Green Supermix (Bio-Rad). The relative mRNA transcription levels of ROS, MMP-2, MMP-9, MMP-13, TIMP-1, TIMP-3, decorin, biglycan, and type I and type III collagen were calculated using the 2- $\Delta \Delta \mathrm{Ct}$ 
method. GAPDH was used as a housekeeping gene to normalize the expression of genes of interest in the present study.

\section{In vivo studies}

\section{Power analysis and study groups}

The animal experimental protocol was approved by the Animal Ethical and Welfare Department of Shanghai Jiaotong University.

A power analysis was performed with $\alpha=0.05,1-\beta=0.8$, and an assumed dropout rate of $25 \%$ to calculate the sample size required for the animal experiments. A sample size of eight shoulders was required to detect a significant difference in the biomechanical analysis of the ultimate load to failure. In addition, we allocated four shoulders for histological analysis in each group. Thus, a total of 36 shoulders (18 male Sprague Dawley rats aged 12 weeks) were included in the present study and divided into the following three groups: saline injection group (control group), dexamethasone injection group (GCs group), and dexamethasone + ASC-Exos injection group (GCs + ASC-Exos group). Each shoulder was randomly chosen to receive an injection of either saline, dexamethasone, or dexamethasone + ASCExos.

\section{Establishment of a chronic rotator cuff injured-intact model and interventions}

After the induction of general anesthesia, a 1-cm longitudinal skin incision was made over the lateral shoulder joint under sterile conditions. The supra-infraspinatus tendon was exposed by splitting the deltoid muscle. The rotator cuff injured-intact model was established by completely cutting the supraspinatus tendon from the greater tubercle to create a full-thickness injury while leaving the infraspinatus tendon intact; this was left for 4 weeks to establish a chronic injured-intact model that simulated the clinical setting. The muscle, subcutaneous tissue, and skin were closed as separate layers.

After 6 weeks, a single dose of saline, dexamethasone $(0.1 \mathrm{mg} / \mathrm{kg})$, or dexamethasone $(0.1 \mathrm{mg} / \mathrm{kg})+$ ASC-Exos $\left(10^{11}\right.$ pellets $\left./ \mathrm{ml}\right)$ of the same volume was injected into the subacromial space in each shoulder. At 1 week after injection, all rats were euthanized by $\mathrm{CO}_{2}$ inhalation. The supra-infraspinatushumerus complex was carefully harvested. Histological and biomechanical analysis were performed after macroscopic evaluation of the harvested sample.

\section{Histological analysis}


Samples were fixed in $4 \%$ buffered paraformaldehyde for 24 hours and embedded in paraffin.

Consecutive 3-um-thick sections were cut parallel to the long axis of the supra-infraspinatus tendon in the coronal plane.

To evaluate the general appearance, sections were stained with hematoxylin and eosin (H\&E). To evaluate collagen degenerations, sections were stained with type I and III collagen antibodies. To evaluate fatty infiltration, sections were stained with oil red 0 .

Images of the entire section of the supra-infraspinatus were acquired by digital slide scanning (Pannoramic MIDI; 3DHISTECH Ltd.). The intensities of type I and type III collagen and fatty infiltration were quantitatively measured using ImageJ software (National Institutes of Health) as previously described[25].

Semi-quantitative analysis was also performed as previously described to comprehensively evaluate the histological properties of the tendon (Table 1)[26].

Table 1

The Tendon Maturing Scoring System

\begin{tabular}{|lcccc|}
\hline & 1 & 2 & 3 & 4 \\
\hline Cellularity & Marked & Moderate & Mild & Minimal \\
\hline Proportion of cells resembling tenocytes & $<25 \%$ & $25-50 \%$ & $50-$ & $>75 \%$ \\
\hline Proportion of parallel cells & $<25 \%$ & $25-50 \%$ & $50-$ & $>75 \%$ \\
\hline Vascularity & Marked & Moderate & Mild & Minimal \\
\hline $\begin{array}{l}\text { Proportion of fibers of large diameter characteristic of } \\
\text { mature tendon fibers }\end{array}$ & $<25 \%$ & $25-50 \%$ & $50-$ & $>75 \%$ \\
\hline Proportion of parallel fibers & $<25 \%$ & $25-50 \%$ & $50-$ & $>75 \%$ \\
\hline
\end{tabular}

\section{Biomechanical analysis}

Before biomechanical testing, dissections were performed to separate the supra-infraspinatus-humerus complex into two parts: the isolated supraspinatus and the infraspinatus-humerus complex. The crosssectional areas of both the supra- and infraspinatus tendons were measured using digital calipers. A custom-designed uniaxial testing machine (Instron 5569, USA) was used to perform the biomechanical analysis. 
To fix the isolated supraspinatus, the muscular end was gripped in a serrated clamp to prevent slippage, and the tendinous end was cross-sutured using No. 2 Orthocord suture (DePuy Mitek) and then tied to the sensor of the device. To fix the infraspinatus-humerus complex, the humerus was mounted in a cylindrical holder using six interference screws, and the muscular end was gripped in a serrated clamp connected to the sensor system. The musculotendinous part of the infraspinatus was placed at an angle of approximately $90^{\circ}$ to the humerus to mimic the anatomic direction of tensile force.

After preloading for 5 minutes using a $0.5 \mathrm{~N}$ tensile force, the sample received 10 cycles of loading ranging from $0.5 \mathrm{~N}$ to $2 \mathrm{~N}$ to minimize the viscoelastic effects. Immediately after preconditioning, the load-to-failure test started with uniaxial tension at $1 \mathrm{~mm} /$ minute. The ultimate load to failure was defined as the first significant decrease in the load-displacement curve, and the stress was calculated by dividing the ultimate load to failure by the initial cross-sectional area. The mode of failure was also recorded.

\section{Statistical analysis}

All data are expressed as mean \pm SD. Statistical analysis was performed using SPSS software (version 15, SPSS). One-way analysis of variance with post hoc testing using the Bonferroni method was carried out. Significance was set at $P<0.05$.

\section{Results}

\section{Characterization of ASC-Exos}

The TEM and NTA revealed spherical vesicles with a particle size distribution of 50 to $150 \mathrm{~nm}$. Westernblotting analysis showed that these vesicles had strong surface expression of exosomal markers, including CD9, CD63, and TSG-101 (Figure 1).

\section{Inflammation analysis}

\section{Cell proliferation}

Dexamethasone treatment significantly decreased the proliferation intensity of rat raw cells to 0.82 -, 0.89 -, and 0.88 -fold at 12,24 , and 48 hours, respectively, compared with the control group.

To our surprise, additional treatment with ASC-Exos did not further decrease the proliferation intensity, but overrode the detrimental effect of dexamethasone on the proliferation of raw cells, with the GCs + ASCExos group showing no significant differences in proliferation intensity compared with the control group (Figure 2A). 


\section{Cell migration}

Dexamethasone treatment significantly decreased the migration of rat raw cells to 0.69 -fold at 24 hours, compared with the control group.

Consistent with the cell proliferation results, additional treatment with ASC-Exos did not further decrease cell migration, but overrode the detrimental effect of dexamethasone on the migration of raw cells, with the GCs + ASC-Exos group showing no significant differences in migration compared with the control group (Figure 2B, 2C).

\section{Secretion of inflammatory-related cytokines}

\section{Pro-inflammatory cytokines}

Dexamethasone treatment significantly downregulated the secretion of TNF- $a$, IL-1 $\mathrm{a}$, and IL-1 $\beta$ from rat raw cells to $0.75-, 0.85$-, and 0.49 -fold, respectively, compared with the control group.

In contrast to the cell proliferation and migration results, additional treatment with ASC-Exos further significantly downregulated the secretion of TNF-a, IL-1a, and IL-1 $\beta$ to $0.54-, 0.76-$, and 0.33-fold, respectively, compared with the control group.

Significant differences were also detected between the GCs group and the GCs + ASC-Exos group, indicating that additional treatment with ASC-Exos might exert a stronger anti-inflammatory effect than dexamethasone alone (Figure 3A, 3B, 3C).

\section{Anti-inflammatory cytokines}

Dexamethasone treatment significantly upregulated the secretion of IL-4 to 1.39-fold, and significantly downregulated the secretion of IL-10 to 0.81 -fold, respectively, compared with the control group.

Additional treatment with ASC-Exos further significantly upregulated the secretion of IL-4 and IL-10 to 3.68- and 2.31-fold, respectively, compared with the control group.

Consistent with the pro-inflammatory cytokine results, significant differences were also detected between the GCs group and the GCs + ASC-Exos group, indicating that additional treatment with ASC-Exos might exert a stronger anti-inflammatory effect than dexamethasone alone (Figure 3D, 3E).

\section{Cytoprotective analysis}




\section{Cell proliferation}

Dexamethasone treatment significantly decreased the proliferation intensity of rat tenocytes to 0.74 -, 0.91 -, and 0.70 -fold at 12,24 , and 48 hours, respectively, compared with the control group.

Additional treatment with ASC-Exos not only overrode the detrimental effect of dexamethasone on the proliferation of rat tenocytes, but further significantly promoted their proliferation intensity to 1.17-, 1.22-, and 1.13-fold at 12, 24, and 48 hours, respectively, compared with the control group (Figure 4A).

\section{Cell migration}

Dexamethasone treatment significantly decreased the migration of rat tenocytes to 0.55 -fold at 24 hours, compared with the control group.

Additional treatment with ASC-Exos not only overrode the detrimental effect of dexamethasone on the migration of rat tenocytes, but further significantly increased their migration to 1.13-fold at 24 hours, compared with the control group (Figure 4B, 4C).

\section{Cell senescence}

Dexamethasone treatment significantly increased the senescence intensity of rat tenocytes to 1.92-fold at 24 hours, compared with the control group.

Additional treatment with ASC-Exos overrode the detrimental effect of dexamethasone on the senescence intensity of rat tenocytes, as shown by the lack of a significant difference in the senescence intensity between the GCs + ASC-Exos group and the control group (Figure 5A, 5B).

\section{Cell apoptosis}

Dexamethasone treatment significantly increased the apoptosis percentage of rat tenocytes to 1.34-fold at 24 hours, compared with the control group.

Additional treatment with ASC-Exos overrode the detrimental effect of dexamethasone on the apoptosis percentage of rat tenocytes, as shown by the lack of a significant difference in the apoptosis percentage between the GCs + ASC-Exos group and the control group (Figure 5C, 5D).

\section{Transcription of ROS}

Dexamethasone treatment significantly upregulated the transcription of ROS of rat tenocytes to 2.40 -fold of rat tenocytes at 72 hours, compared with the control group. 
Additional treatment with ASC-Exos not only overrode the effect of dexamethasone on the ROS transcription by rat tenocytes, but further significantly decreased their transcription to 0.41 -fold at 72 hours, compared with the control group (Figure 6A).

\section{Transcription of degradative enzymes and their inhibitors}

Dexamethasone treatment significantly upregulated the transcription of MMP-2, MMP-9, and MMP-13 by rat tenocytes to 1.79-, 2.33-, and 3.62-fold, respectively, compared with the control group. Additional treatment with ASC-Exos overrode the effect of dexamethasone on the transcription of degradative enzymes of rat tenocytes, as shown by the lack of significant difference between the GCs + ASC-Exos group and the control group.

Dexamethasone treatment had little effect on the transcription of degradative enzyme inhibitors by rat tenocytes, as shown by the lack of significant difference between the GCs group and the control group regarding the transcription of TIMP-1 and TIMP-3. Additional treatment with ASC-Exos upregulated the transcription of TIMP-1 and TIMP-3 of rat tenocytes to 3.95- and 2.72-fold at 72 hours, respectively, compared with the control group (Figure 6B-6F).

\section{Transcription of tenocytic matrix molecules}

Dexamethasone treatment significantly downregulated the transcription of decorin and biglycan by rat tenocytes to 0.65 - and 0.53 -fold at 72 hours, respectively, compared with the control group. Additional treatment with ASC-Exos not only overrode the effect of dexamethasone on the decorin and biglycan transcription by rat tenocytes, but further significantly increased their transcription to 2.41 - and 1.40 -fold at 72 hours, respectively, compared with the control group (Figure 7A, 7B).

Dexamethasone treatment significantly downregulated the transcription of type I collagen by rat tenocytes to 0.27 -fold at 72 hours compared with the control group. Transcription of type III collagen was also downregulated in the GCs group to 0.78-fold compared with the control group, but this difference did not reach statistical significance. Additional treatment with ASC-Exos not only overrode the effect of dexamethasone on the transcription of type I and type III collagen by rat tenocytes, but further significantly increased their transcription to 4.90 - and 3.23-fold at 72 hours, respectively, compared with the control group (Figure 7C, 7D).

Dexamethasone treatment also significantly downregulated the type I/III transcription ratio to 0.34 -fold compared with the control group. Additional treatment with ASC-Exos not only overrode the effect of dexamethasone on the type I/III transcription ratio of rat tenocytes, but further significantly increased their transcription ratio to 1.52 -fold at 72 hours compared with the control group (Figure 7E).

Macroscopic evaluation of the supra-infraspinatus-humerus complex 
The torn supraspinatus tendon was connected to the humerus through fibrous tissue, and the connection between the infraspinatus tendon and the humerus remained complete. There was no evidence of infection.

\section{Histological analysis}

\section{Injured supraspinatus tendon}

Generally, increased cellularity, vascularity, thinning, separation, and disorganization of collagen fibers, and fatty infiltration were observed in all three groups. These signs were most significant in the GCs group (Figure 8).

Quantitatively, the GCs group exhibited significantly higher fatty infiltration, and severe collagen degeneration (significantly lower type I collagen and higher type III collagen density) than both the control and GCs + ASC-Exos group. The immunohistochemical variables did not significantly differ between the control and GCs + ASC-Exos groups (Figure 9A, 9B, 9C).

Semi-quantitative analysis results showed that the histological properties in the GCs group were significantly worse than those in the control and GCs + ASC-Exos groups. There were no significant differences between the control and GCs + ASC-Exos groups (Figure 9D).

\section{Intact infraspinatus tendon}

Generally, slightly increased cellularity, vascularity, thinning, separation, and disorganization of collagen fibers were observed in all three groups. These signs were relatively most significant in the GCs group. There was no evidence of fatty infiltration in any of the three groups (Figure 10).

Quantitatively, the GCs group exhibited severe collagen degeneration (significantly lower type I collagen and higher type III collagen density) than both the control and GCs + ASC-Exos groups. There were no significant differences between the control and GCs + ASC-Exos groups with regard to collagen degeneration (Figure 11A, 11B).

Semi-quantitative analysis results showed that the histological properties in the GCs group were significantly worse than those in the control and GCs + ASC-Exos groups. There were no significant differences between the control and GCs+ ASC-Exos groups (Figure 11C).

\section{Biomechanical analysis}

\section{Injured supraspinatus tendon}


The cross-sectional area of the supraspinatus tendon did not significantly differ between the three groups (Figure 12A).

The GCs injection significantly decreased the ultimate load to failure and the ultimate stress to failure to 0.66- and 0.71-fold, respectively, compared with the control group (Figure 12B, 12C). Concomitant injection of ASC-Exos and GCs overrode the GCs-induced detrimental effects on the biomechanical properties of the injured supraspinatus tendon, as shown by the lack of significant differences between the GCs + ASC-Exos group and the control group in the ultimate load to failure and the ultimate stress to failure (Figure 12B, 12C).

All specimens failed at the middle of the tendon.

\section{Intact infraspinatus tendon}

The cross-sectional area of the intact infraspinatus tendon did not significantly differ between the three groups (Figure 13A).

The GCs injection significantly decreased the ultimate load to failure and the ultimate stress to failure to 0.64- and 0.74-fold, respectively, compared with the control group (Figure 13B, 13C). Concomitant injection of ASC-Exos and GCs overrode the GCs-induced detrimental effects on the biomechanical properties of the intact infraspinatus tendon, as shown by the lack of significant differences between the GCs + ASC-Exos group and the control group in the ultimate load to failure and the ultimate stress to failure (Figure 13B, 13C).

All specimens failed at the tendon-to-bone interface.

\section{Discussion}

The most important finding of the present study was that additional treatment with ASC-Exos not only facilitated the anti-inflammatory effect of GCs, but overrode the negative effect of GCs on tenocytes, alleviating the detrimental effect of a GCs injection on an injured-intact rotator cuff. The in vitro results showed that additional treatment with ASC-Exos and GCs exerted a synergic anti-inflammatory effect mainly by suppressing the secretion of pro-inflammatory cytokines and increasing the secretion of antiinflammatory cytokines from raw cells; the cytoprotective effect of ASC-Exos on tenocytes was achieved mainly by suppressing the GCs-induced transcription of ROS and MMPs, and increasing the transcription of TIMPs and GCs-suppressed tenocytic matrix molecules (including decorin and biglycan) and the type $\mathrm{I} / \mathrm{III}$ collagen ratio. The analysis of the rat model of chronic rotator cuff tear further showed that the detriments on both the histological and mechanical properties of the injured-intact rotator cuff induced by a single GCs injection at 1 week were restored by an additional ASC-Exos injection.

Subacromial GCs injections are commonly performed to treat multiple shoulder pathologic conditions to reduce pain and improve range of motion[3-5, 8]. However, a major concern limiting the further use of 
such GCs injection is the adverse effects that accelerate tendon degeneration $[2,6,7,15,18]$. Nowadays, it is commonly accepted that subacromial GCs injections lead to a significant decrease in the mechanical properties of both injured and normal rotator cuff tendons[2, 6, 15]. Inspired by the bioactive effect of platelet-rich plasma (PRP), an autologous blood plasma concentrate that contains various growth factors, Jo et al. investigated the effect of concomitant treatment with PRP and GCs on tenocytes in a degenerative condition induced by IL-1 $\beta[7]$. Their results showed that PRP produced a synergic antiinflammatory effect with GCs and antagonized their adverse effects[7]. In vitro evidence from other studies has also showed that some bioactive factors have a cytoprotective effect on tenocytes against GCs-induced cellular senescence and apoptosis[27-29]. However, these previous studies only evaluated the anti-inflammatory effect with or without cytoprotective effects in vitro rather than in vivo.

Macrophages play a key role during the inflammatory infiltration process after initial rotator cuff injury to cause shoulder pain, degeneration, and dysfunction[11-14, 30]. During the early inflammatory infiltration stage, macrophages cause damage to normal tissues when phagocytizing and clearing necrotic tissues[11-13, 30]; during the inflammatory regression stage, residual lipid-laden macrophages differentiate into adipocytes, resulting in fatty accumulation in rotator cuff muscles[31]. Furthermore, multiple pro-inflammatory cytokines secreted by macrophages start the inflammatory infiltration cascade that leads to the secondary damage[11-13,30]. Thus, the main therapeutic objective to improve impaired shoulder function is to inhibit macrophage proliferation, migration, and secretion of pro-inflammatory cytokines. In the present study, a single GCs injection significantly decreased rat raw cell proliferation, migration, secretion of pro-inflammatory cytokines including TNF-a, IL-1a, and IL-1 $\beta$, and increased the secretion of the anti-inflammatory cytokine IL-4. Additional treatment with ASC-Exos plus GCs further significantly decreased the secretion of pro-inflammatory cytokines and increased secretion of antiinflammatory cytokines including IL-4 and IL-10, suggesting a stronger synergic anti-inflammatory effect. However, this synergic anti-inflammatory effect was not achieved by further decreasing the proliferation and migration of rat raw cells, as the proliferation and migration intensity in the GCs + ASC-Exos group were not different to the control group. We considered that this synergic anti-inflammatory phenomenon might be achieved by the ASC-Exos-driven M2 phenotypic transformation of macrophages. Recent studies from Chen et al. found that a medium conditioned with human bone marrow-derived stem cells, which could be regarded as exosomes from bone marrow-derived stem cells, had a significant impact on macrophage polarization by inhibiting M1 phenotype polarization and promoting M2 phenotype polarization[12]. Zhang et al. also reported that MSC-Exos enhance the survival of cartilage grafts via the phenotypic transformation of macrophages from M1 to M2[23]. The results from these previous studies indicate that the synergic anti-inflammatory effect obtained after additional treatment with ASC-Exos plus GCs in the present study might be achieved by regulating the phenotypic transformation of macrophages. Further studies are needed to investigate this phenomenon.

Additional treatment with ASC-Exos plus GCs not only exerted a stronger synergic anti-inflammatory effect than GCs treatment alone, but also restored the GCs-induced detrimental effects on tenocytes. The generation of ROS is reportedly a sensitive indicator of GCs-induced mitochondrial detriment, resulting in decreased cell proliferation and migration, and increased cell senescence and apoptosis[18]. In the 
present study, additional treatment with ACS-Exos significantly reduced the GCs-induced ROS transcription, decreasing the senescence and apoptosis, and increasing the proliferation and migration of tenocytes. MMPs are responsible for the degradation of the extracellular matrix, while this process is antagonized by TIMPs during tendon development, morphogenesis, and normal remodeling[26, 32, 33]. Although the roles of MMPs and TIMPs may be either pathological or physiological, the present results suggest that additional treatment with ASC-Exos somewhat decreased the GCs-induced MMP level and increased the TIMP level, showing the possible role of ASC-Exos in maintaining the metabolic homeostasis of tendons[26, 32, 33]. Increased amount of type III collagen is regarded as a repair response to tissue injury and formation of wound bed granulation tissue, while successful remodeling is characterized by the replacement of type III collagen with type I collagen in the healing of torn rotator cuff tendons [26,32]. The present results show that GCs treatment significantly decreased the type I/III collagen transcription ratio, which might result in a poorly organized tendon, while additional treatment with ASC-Exos was able to restore that adverse effect. Decorin and biglycan are also major components of leucine-rich proteoglycan in tendons[22]. Additional treatment with ASC-Exos significantly increased the GCs-suppressed transcription of decorin and biglycan, further indicating that ASC-Exos had a positive effect on tenocytic differentiation.

Our hypothesis was further supported by in vivo studies that showed that an additional injection of ASCExos significantly improved both the histological and mechanical properties of GCs-induced detriments on rotator cuff tendons. Consistent with our in vitro studies, the disorganization, collagen degenerative changes from type I to type III, fatty infiltration, and compromised tendon mechanical properties induced by a GCs injection were restored by an additional injection of ASC-Exos. The potential benefits underlying the contribution of ASC-Exos to synergic anti-inflammatory effects and antagonizing the detrimental effects of GCs might be attributable to stem cell-derived exosome-regulated immunity and growth effects[20, 22].

Published data concerning the protection against GCs-induced tendon damage are limited. Poulsen et al. investigated GCs-induced detriments of tenocytes and found that vitamin $\mathrm{C}$ and insulin antagonize the GCs-induced inhibition of the ERK and Akt signaling pathways as well as the GCs-induced increased level of FOXO, restoring normal cell proliferation and collagen synthesis in GCs-treated tenocytes[18]. These previous results identify the signaling pathways underlying the GCs-induced detrimental effects on tenocytes, and the authors suggested potential strategies against the adverse effects of GCs.

The present study had the following limitations. First, although we have shown that ASC-Exos exerted a synergic anti-inflammatory effect with GCs and override their detrimental effects on the rotator cuff, but we did not perform further investigations to elaborate this phenomenon and reveal underlying mechanism, which is worth investigating in our future studies. Second, we used normal rather than degenerative tenocytes in the in vitro study, and the results might differ under different conditions. Third, we only investigated the effect of a single GCs injection with or without ASC-Exos on the rotator cuff tendons. As previous studies have showed that the detrimental effect of a single GCs injection on tendons is transient, both the histological and mechanical properties of the GCs-treated tendon may 
return to normal levels by 3 weeks[2]. Thus, the effect on the rotator cuff of repeated GCs injections with or without ASC-Exos is worth investigating in the future. Fourth, the anti-inflammatory and analgesic effects are the main areas of interest regarding GCs injections in clinical practice. However, it is difficult to evaluate changes in pain levels after GCs injections in an animal model, and this important aspect was lacking in the present study. However, the present findings have substantial implications for clinical application. As GCs injections lead to a significant decrease in the mechanical properties of tendons, repeated GCs injection is avoided in clinical practice. The present study found that concomitant treatment with GCs and ASC-Exos not only exerted a stronger synergic anti-inflammatory effect, but also restored the GCs-induced detrimental effects on tendons. This might be a possible strategy for repeated GCs injections in the future.

\section{Conclusion}

The combination of GCs and ASC-Exos exerted a stronger anti-inflammatory effect than GCs alone and overrode the detrimental effects of GCs on the rotator cuff.

\section{Abbreviations}

GCs: glucocorticoids

ASC-Exos: adipose stem cell-derived exosomes

TEM: transmission electron microscopy

NTA: nanoparticle tracking analysis

FBS: fetal bovine serum

ELISA: enzyme linked immunosorbent assay

Rt-PCR: real-time polymerase chain reaction

HE: hematoxylin and eosin

PRP: platelet-rich plasma

\section{Declarations}

\section{Ethics approval and consent to participate}

The animal experimental protocol was approved by the Animal Ethical and Welfare Department of Shanghai Jiaotong University. 


\section{Consent for publication}

Not applicable.

\section{Availability of data and materials}

The datasets used a during the current study are available from the corresponding author on reasonable request.

\section{Competing interests}

The authors have declared that no competing interest exists

\section{Funding}

This study was supported by The New Frontier Multi-center Project of Shenkang Hospital Development Center of Shanghai (Grant No. SHDC12017121), (China) State's Key Project of Research and Development Plan (Grant No. 2018YFF0300504), (China) State's Key Project of Research and Development Plan (Grant No. 2018YFC110620), the National Natural Science Foundation of China (Grant No. 82072426), and the National Natural Science Foundation of China (Grant No. 81871753).

\section{Authors' contributions}

X.Z. and A.L.: Conception and design and manuscript writing. K.H., H.Z., and X.H.: Data collection, analysis and interpretation. J.H, J.J., and J.Z.: Provision of study material and final approval of manuscript

\section{Acknowledgements}

Not applicable.

\section{References}

1. Ostor, Jk A. Diagnosis and relation to general health of shoulder disorders presenting to primary care. Rheumatology. 2005;(6):800-805.

2. Mikolyzk DK, Wei AS, Tonino P, Marra G, Williams DA, Himes RD, Wezeman FH, Callaci JJ. Effect of corticosteroids on the biomechanical strength of rat rotator cuff tendon. J Bone Joint Surg Am. 2009;91(5):1172-1180. 
3. Bartolozzi A, Andreychik D, Ahmad S. Determinants of outcome in the treatment of rotator cuff disease. Clin Orthop Relat Res. 1994;308(308):90-97.

4. Breazeale NM, Craig EV. Partial-thickness rotator cuff tears - Pathogenesis and treatment. Orthopedic Clinics of North America. 1997;28(2):145-155.

5. Fukuda, Hiroaki. Partial-thickness rotator cuff tears: a modern view on Codman's classic. Journal of Shoulder \& Elbow Surgery. 2000;9(2):163-168.

6. Haraldsson BT, Langberg H, Aagaard P, Zuurmond AM, van El B, Degroot J, Kjaer M, Magnusson SP. Corticosteroids reduce the tensile strength of isolated collagen fascicles. Am J Sports Med. 2006;34(12):1992-1997.

7. Jo CH, Lee SY, Yoon KS, Shin S. Effects of Platelet-Rich Plasma With Concomitant Use of a Corticosteroid on Tenocytes From Degenerative Rotator Cuff Tears in Interleukin 1beta-Induced Tendinopathic Conditions. Am J Sports Med. 2017;45(5):1141-1150.

8. Arroll B, Goodyear-Smith F. Corticosteroid injections for painful shoulder: A meta-analysis. British Journal of General Practice. 2005;55(512):224-228.

9. Doomsgoossens A. Sensitisation to corticosteroids. Consequences for anti-inflammatory therapy. Drug Saf. 1995;13(2):123-129.

10. Granier M, Massin F, Pasquie JL. Pro- and Anti-Arrhythmic Effects of Anti-Inflammatory Drugs. Antiinflamm Antiallergy Agents Med Chem. 2013;12(1):-.

11. Philippou A, Maridaki M, Theos A, Koutsilieris M. Cytokines in muscle damage. Adv Clin Chem. 2012;58:49-87.

12. Chen W, Sun Y, Gu X, Cai J, Liu X, Zhang X, Chen J, Hao Y, Chen S. Conditioned medium of human bone marrow-derived stem cells promotes tendon-bone healing of the rotator cuff in a rat model. Biomaterials. 2021;271:120714.

13. Dakin S, Martinez, FO, Yapp, C, et al. Inflammation activation and resolution in human tendon disease. Science translational medicine. 2015;7(311):311ra173-311ra173.

14. Ji JH, Kim YY, Patel K, Cho N, Park SE, Ko MS, Park SJ, Kim JO. Dexamethaone Facilitates NFkappaB Signal Pathway in TNF-alpha Stimulated Rotator Cuff Tenocytes. J Microbiol Biotechnol. 2019;29(2):297-303.

15. Maman E, Yehuda C, Pritsch T, Morag G, Brosh T, Sharfman Z, Dolkart O. Detrimental Effect of Repeated and Single Subacromial Corticosteroid Injections on the Intact and Injured Rotator Cuff: A Biomechanical and Imaging Study in Rats. Am J Sports Med. 2016;44(1):177-182.

16. Kleinman M, Gross AE. Achilles tendon rupture following steroid injection. Report of three cases. The Journal of Bone and Joint Surgery. 1984;65(9):1345-1347.

17. Rokito AS, Mclaughlin JA, Gallagher MA, Zuckerman JD. Partial rupture of the distal biceps tendon. Journal of Shoulder \& Elbow Surgery. 2001;10(1):377-379.

18. Poulsen RC, Carr AJ, Hulley PA. Protection against glucocorticoid-induced damage in human tenocytes by modulation of ERK, Akt, and forkhead signaling. Endocrinology. 2011;152(2):503-514. 
19. Guo SC, Tao SC, Yin WJ, Qi X, Yuan T, Zhang CQ. Exosomes derived from platelet-rich plasma promote the re-epithelization of chronic cutaneous wounds via activation of YAP in a diabetic rat model. Theranostics. 2017;7(1):81-96.

20. Sevivas N, Teixeira FG, Portugal R, Araujo L, Carrico LF, Ferreira N, Vieira da Silva M, EspregueiraMendes J, Anjo S, Manadas B et al. Mesenchymal Stem Cell Secretome: A Potential Tool for the Prevention of Muscle Degenerative Changes Associated With Chronic Rotator Cuff Tears. Am J Sports Med. 2017;45(1):179-188.

21. Tao SC, Yuan T, Zhang YL, Yin WJ, Guo SC, Zhang CQ. Exosomes derived from miR-140-5poverexpressing human synovial mesenchymal stem cells enhance cartilage tissue regeneration and prevent osteoarthritis of the knee in a rat model. Theranostics. 2017;7(1):180-195.

22. Sevivas N, Teixeira FG, Portugal R, Direito-Santos B, Espregueira-Mendes J, Oliveira FJ, Silva RF, Sousa N, Sow WT, Nguyen LTH et al. Mesenchymal Stem Cell Secretome Improves Tendon Cell Viability In Vitro and Tendon-Bone Healing In Vivo When a Tissue Engineering Strategy Is Used in a Rat Model of Chronic Massive Rotator Cuff Tear. Am J Sports Med. 2018;46(2):449-459.

23. Zhang S, Chuah SJ, Lai RC, Hui JHP, Lim SK, Toh WS. MSC exosomes mediate cartilage repair by enhancing proliferation, attenuating apoptosis and modulating immune reactivity. Biomaterials. 2018;156:16-27.

24. Zhang S, Teo KYW, Chuah SJ, Lai RC, Lim SK, Toh WS. MSC exosomes alleviate temporomandibular joint osteoarthritis by attenuating inflammation and restoring matrix homeostasis. Biomaterials. 2019;200:35-47.

25. Wang C, Song W, Chen B, Liu X, He Y. Exosomes Isolated From Adipose-Derived Stem Cells: A New Cell-Free Approach to Prevent the Muscle Degeneration Associated With Torn Rotator Cuffs. Am J Sports Med. 2019;47(13):3247-3255.

26. Zhang X, Cai Z, Wu M, Huangfu X, Li J, Liu X. Adipose Stem Cell-Derived Exosomes Recover Impaired Matrix Metabolism of Torn Human Rotator Cuff Tendons by Maintaining Tissue Homeostasis. Am J Sports Med. 2021;49(4):899-908.

27. Carofino B, Chowaniec DM, Mccarthy MB, Bradley JP, Delaronde S, Beitzel K, Cote MP, Arciero RA, Mazzocca AD. Corticosteroids and Local Anesthetics Decrease Positive Effects of Platelet-Rich Plasma: An In Vitro Study on Human Tendon Cells. Arthroscopy the Journal of Arthroscopic \& Related Surgery. 2012;28(5):711-719.

28. Beitzel K, Mccarthy MB, Cote MP, Apostolakos J, Russell RP, Bradley J, Elattrache NS, Romeo AA, Arciero RA, Mazzocca AD. The Effect of Ketorolac Tromethamine, Methylprednisolone, and PlateletRich Plasma on Human Chondrocyte and Tenocyte Viability. Arthroscopy the Journal of Arthroscopic \& Related Surgery. 2013;29(7):1164-1174.

29. Muto T, Kokubu T, Mifune Y, Sakata R, Nagura I, Nishimoto H, Harada Y, Nishida K, Kuroda R, Kurosaka M. Platelet-rich plasma protects rotator cuff-derived cells from the deleterious effects of triamcinolone acetonide. Journal of Orthopaedic Research Official Publication of the Orthopaedic Research Society. 2013.31(6):976-982. 
30. Connizzo BK, Grodzinsky AJ. Release of pro-inflammatory cytokines from muscle and bone causes tenocyte death in a novel rotator cuff in vitro explant culture model. Connect Tissue Res. 2018;59(5):423-436.

31. Mendias CL, Roche SM, Harning JA, Davis ME, Lynch E, Enselman E, Jacobson JA, Claflin DR, Calve $S$, Bedi A. Reduced muscle fiber force production and disrupted myofibril architecture in patients with chronic rotator cuff tears. Journal of Shoulder and Elbow Surgery. 2015.

32. Cross JA, Cole BJ, Spatny KP, Sundman E, Romeo AA, Nicholson GP, Wagner B, Fortier LA. LeukocyteReduced Platelet-Rich Plasma Normalizes Matrix Metabolism in Torn Human Rotator Cuff Tendons. Am J Sports Med. 2015;43(12):2898-2906.

33. De GS, Saracino M, Castagna A. Degenerative disease in rotator cuff tears:

\section{Figures}

A

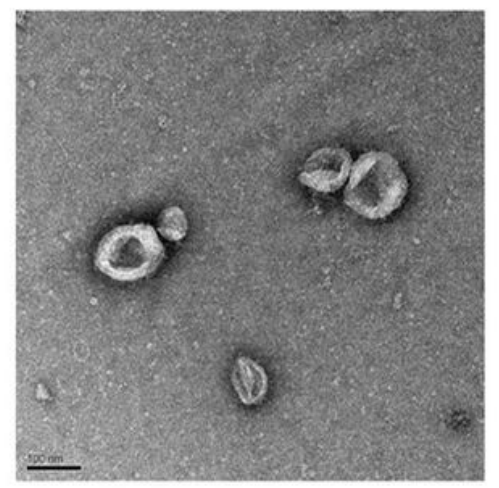

B

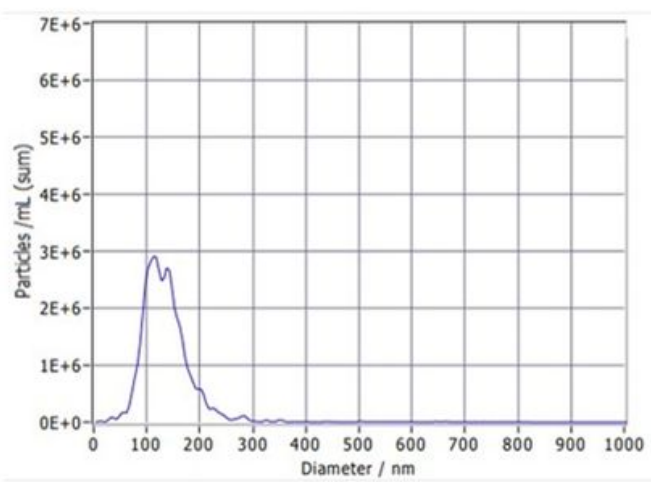

C

CD9

CD63

TGS-101
ASCs ASC-Exos

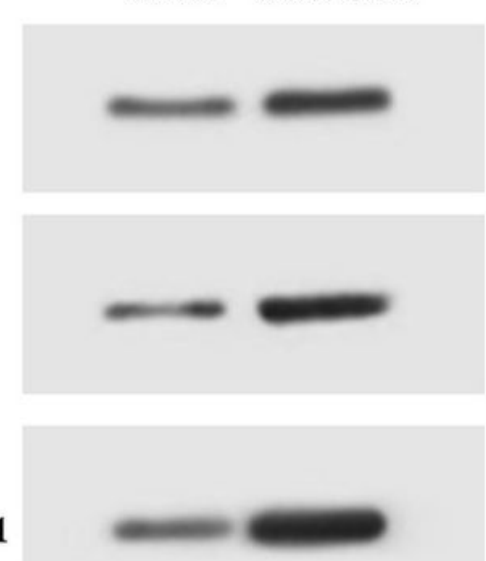

Figure 1

Identification of ASC-Exos. (A) The morphology of ASC-Exos was observed via TEM. (B) The particle size distribution was evaluated using NTA. (C) Exosome surface markers including CD9, CD63, and TSG-101 were evaluated using western-blotting. 


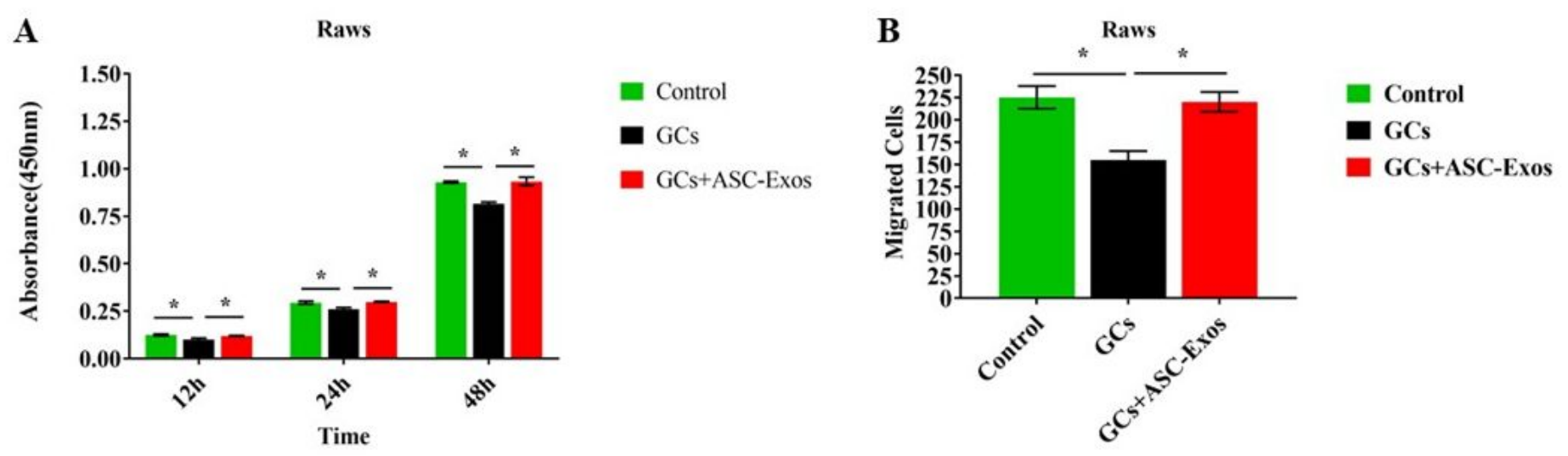

C

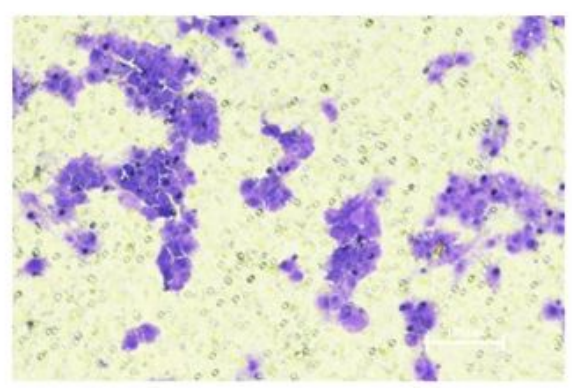

Control

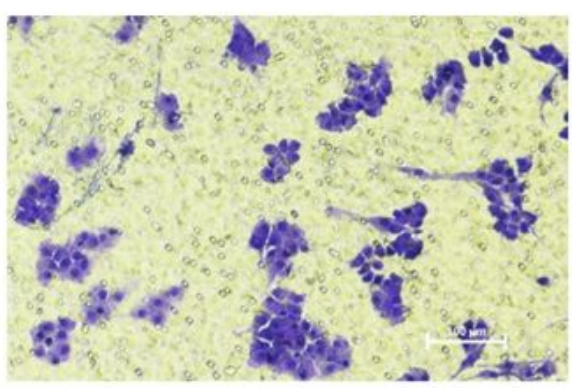

GCs

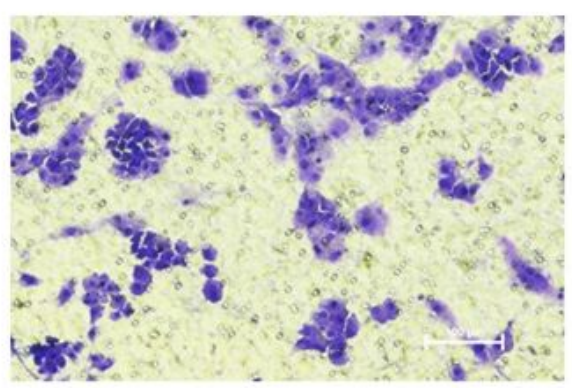

GCs + ASC-Exos

Figure 2

(A) Statistical comparation of rat raw cells proliferation intensity in the saline treated group (Control), GCs treated group (GCs), and GCs + ASC-Exos treated group (GCs + ASC-Exos). (B)Statistical comparation of rat raw cells migration activity in the saline treated group (Control), GCs treated group (GCs), and GCs + ASC-Exos treated group (GCs + ASC-Exos). (C) Representative figures of rat raw cells migration activity in the saline treated group (Control), GCs treated group (GCs), and GCs + ASC-Exos treated group (GCs + ASC-Exos). Data are expressed as mean \pm SD. * indicates significant differences, $P<0.05$. 


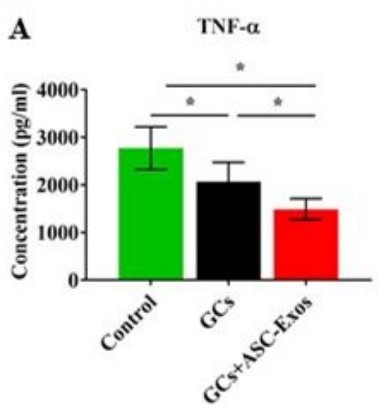

B
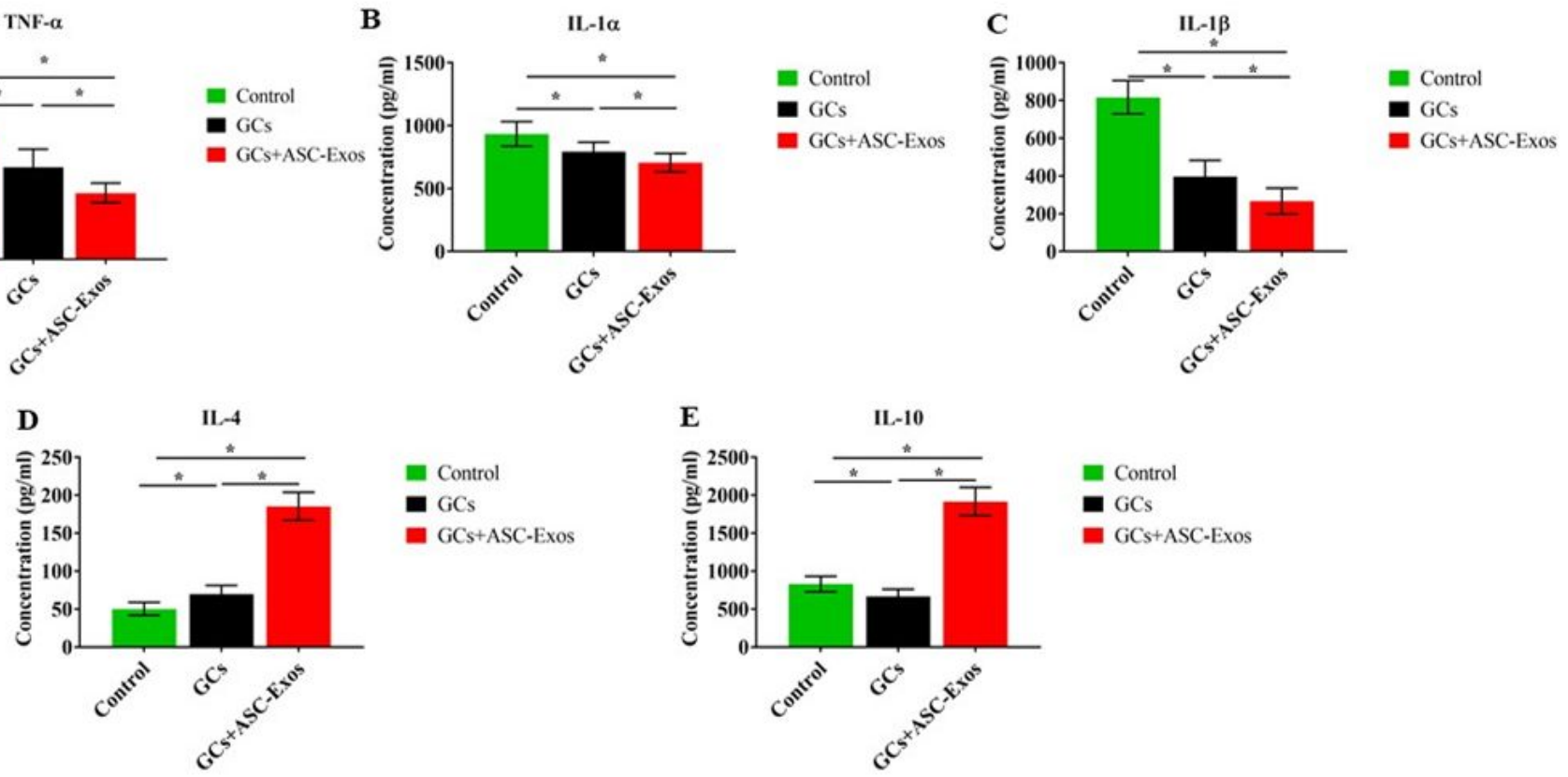

\section{Figure 3}

Statistical comparation of ELISA results of pro-inflammatory cytokines including (A) TNF-a, (B) IL-1a, and (C) IL-1 $\beta$, and anti-inflammatory cytokines including (D) IL-4 and (E) IL-10 secreted from rat raw cells in the saline treated group (Control), GCs treated group (GCs), and GCs + ASC-Exos treated group (GCs + ASC-Exos). Data are expressed as mean $\pm \mathrm{SD}$. * indicates significant differences, $\mathrm{P}<0.05$. 
A

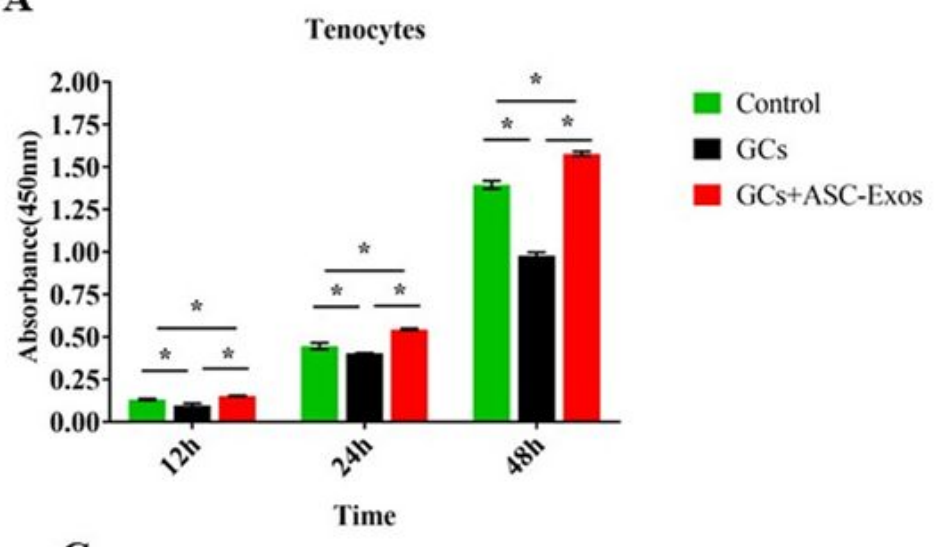

C

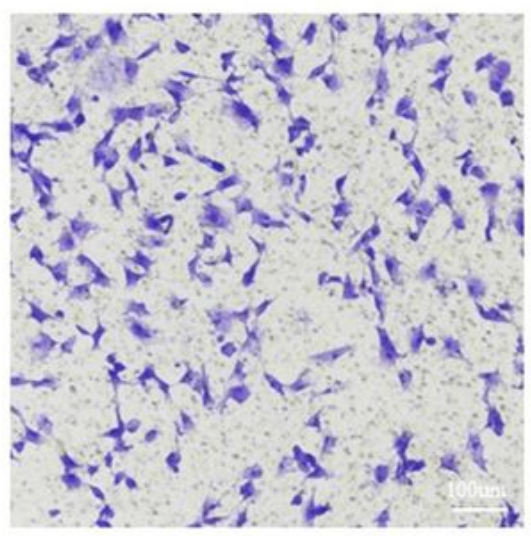

Control

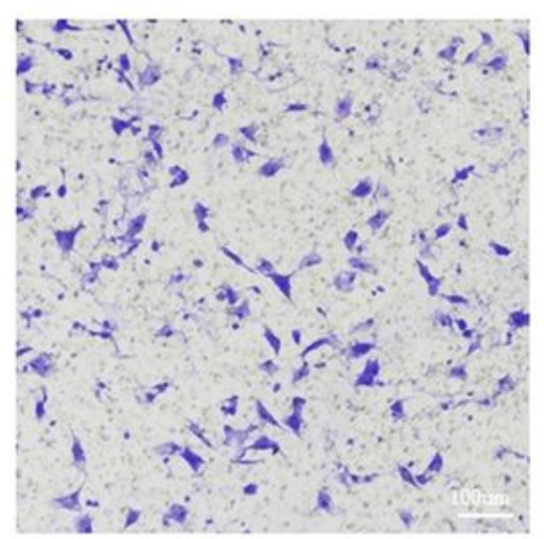

GCs
B
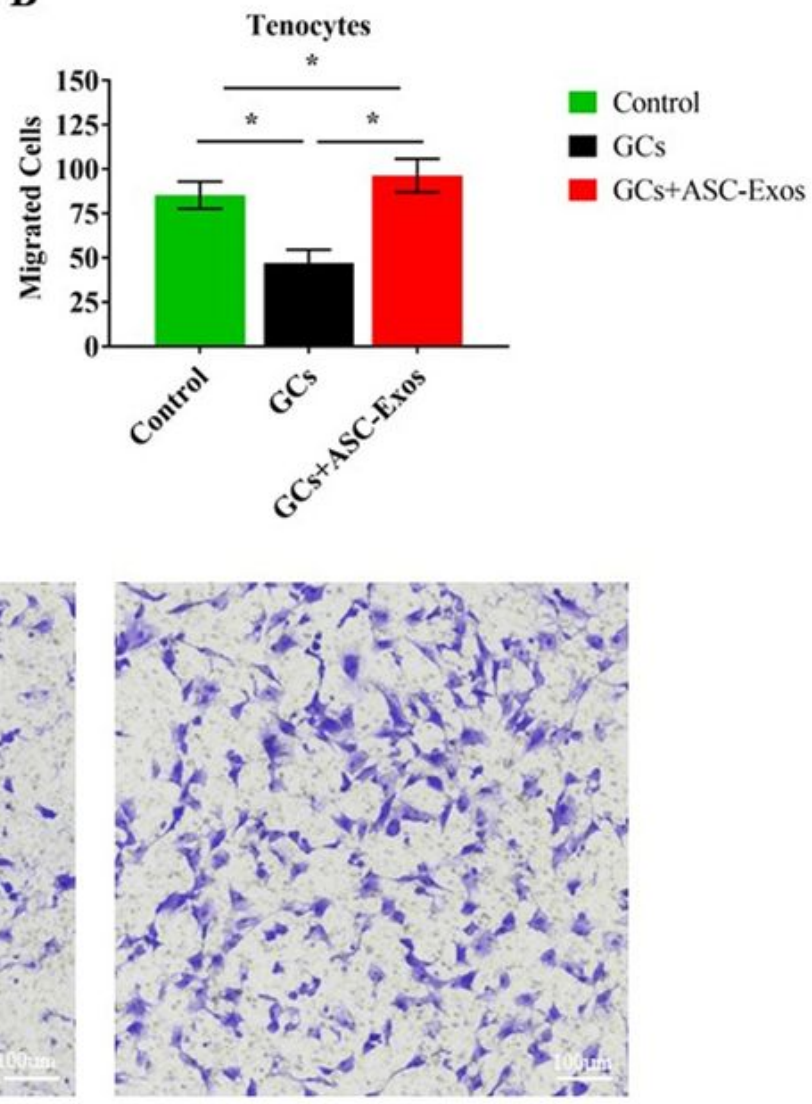

GCs + ASC-Exos

\section{Figure 4}

(A) Statistical comparation of rat tenocytes proliferation intensity in the saline treated group (Control), GCs treated group (GCs), and GCs + ASC-Exos treated group (GCs + ASC-Exos). (B)Statistical comparation of rat tenocytes migration activity in the saline treated group (Control), GCs treated group (GCs), and GCs + ASC-Exos treated group (GCs + ASC-Exos). (C) Representative figures of rat tenocytes migration activity in the saline treated group (Control), GCs treated group (GCs), and GCs + ASC-Exos treated group (GCs + ASC-Exos). Data are expressed as mean \pm SD. * indicates significant differences, $P<0.05$. 


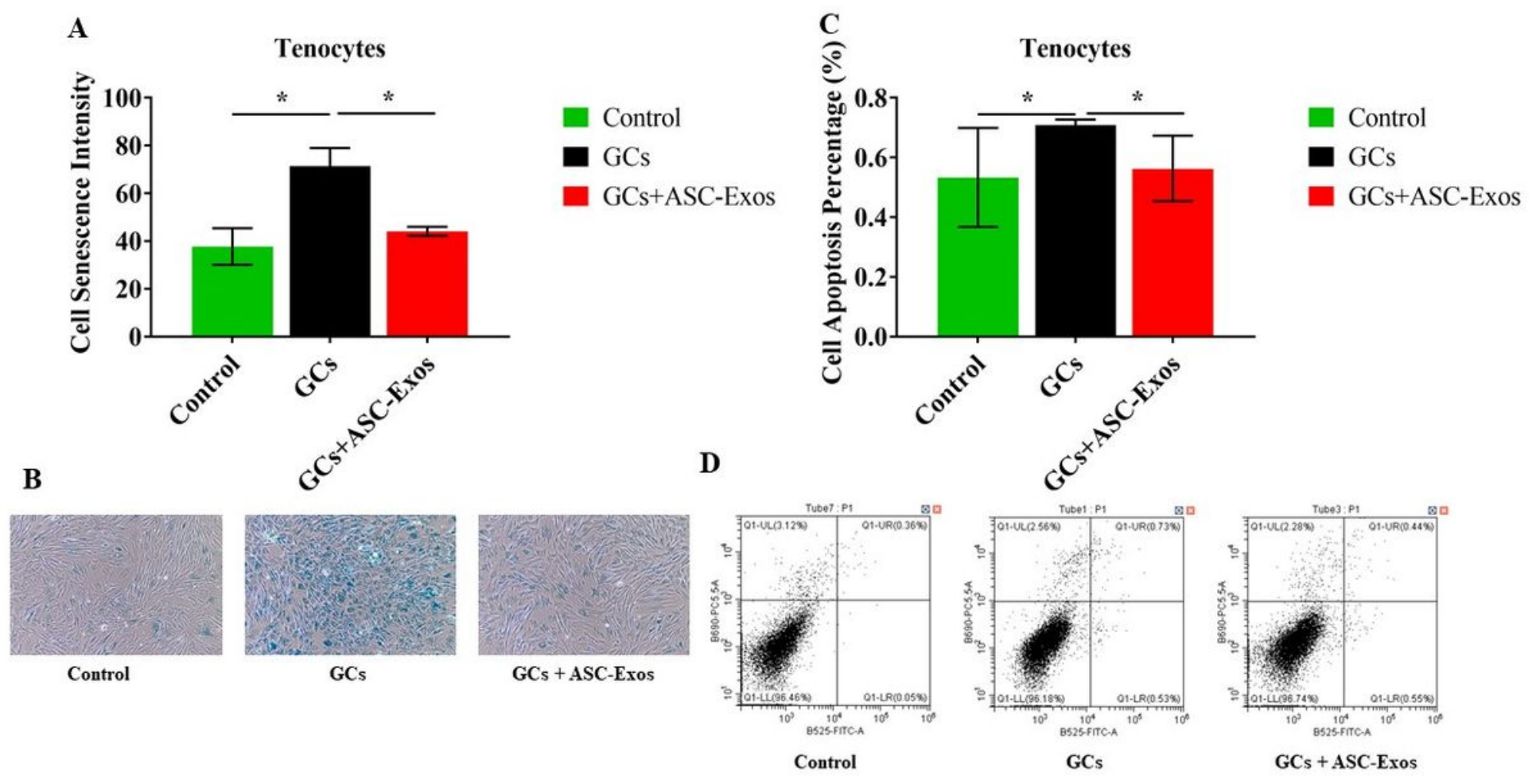

Figure 5

(A) Statistical comparation of rat tenocytes senescence intensity in the saline treated group (Control), GCs treated group (GCs), and GCs + ASC-Exos treated group (GCs + ASC-Exos). (B) Representative figures of $\beta$-Galactosidase staining in the saline treated group (Control), GCs treated group (GCs), and GCs + ASC-Exos treated group (GCs + ASC-Exos). (C) Statistical comparation of rat tenocytes apoptosis percentage in the saline treated group (Control), GCs treated group (GCs), and GCs + ASC-Exos treated group (GCs + ASC-Exos). (D) Representative figures of rat tenocytes apoptosis evaluated using a flow cytometer in the saline treated group (Control), GCs treated group (GCs), and GCs + ASC-Exos treated group (GCs + ASC-Exos). Data are expressed as mean \pm SD. * indicates significant differences, $P<0.05$. 


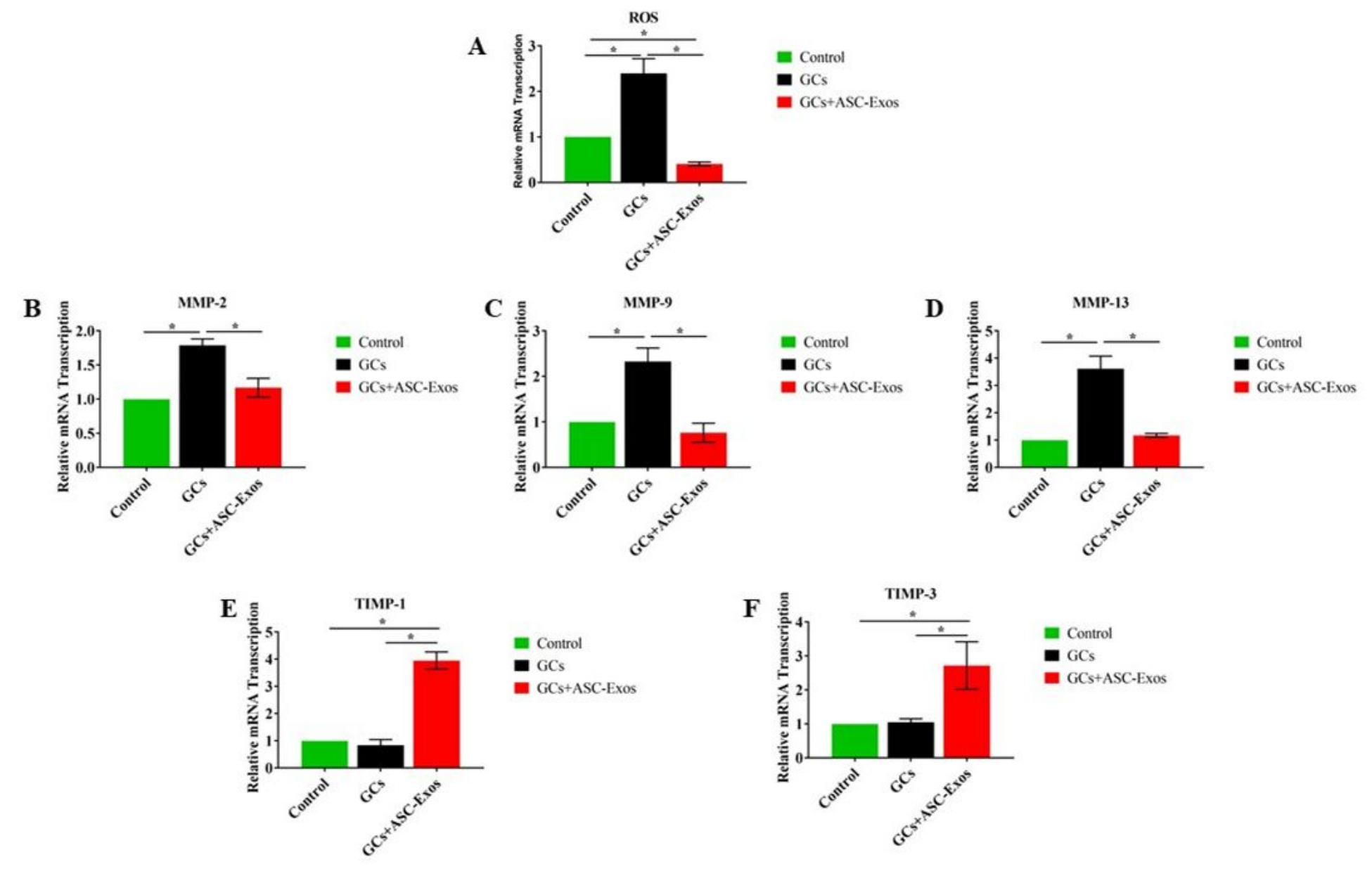

Figure 6

Statistical comparation of rat tenocytes PCR results of (A) ROS, degradative enzymes including (B) MMP2, (C) MMP-9, and (D) MMP-13, and their inhibitors including (E)TIMP-1 and (F) TIMP-3 in the saline treated group (Control), GCs treated group (GCs), and GCs + ASC-Exos treated group (GCs + ASC-Exos). Data are expressed as mean \pm SD. * indicates significant differences, $P<0.05$. 
A

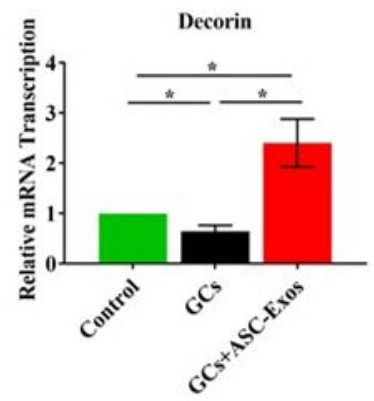

Control

aCs

GCs+ASC-Exos
B

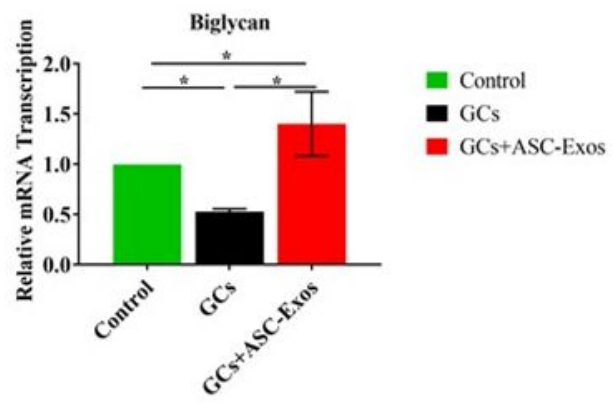

C

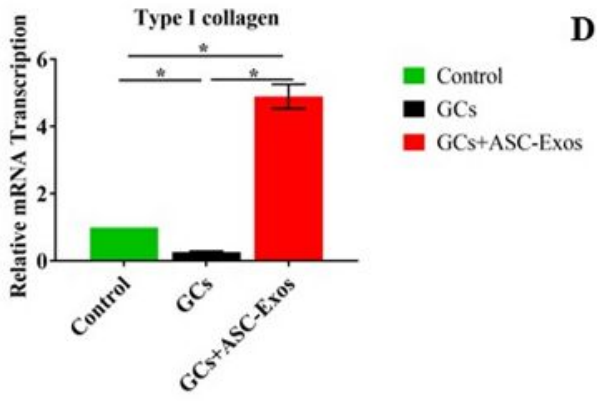

D

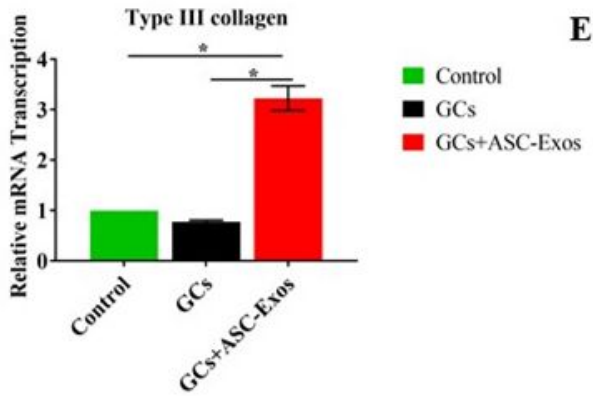

E

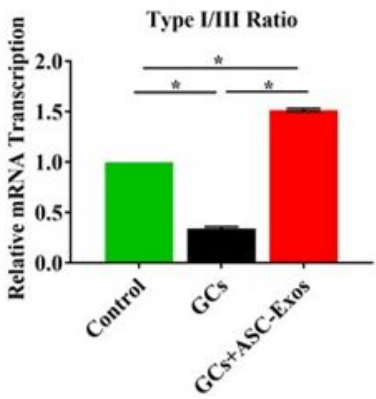

Control

- $\mathrm{GCs}$

- GCs+ASC-Exos

Figure 7

Statistical comparation of rat tenocytes PCR results of tenocytic matrix molecules including (A) Decorin, (B) Biglycan, (C) Type I collagen, (D) Type III collagen, and (E) Type I/III ratio in the saline treated group (Control), GCs treated group (GCs), and GCs + ASC-Exos treated group (GCs + ASC-Exos). Data are expressed as mean \pm SD. * indicates significant differences, $P<0.05$. 


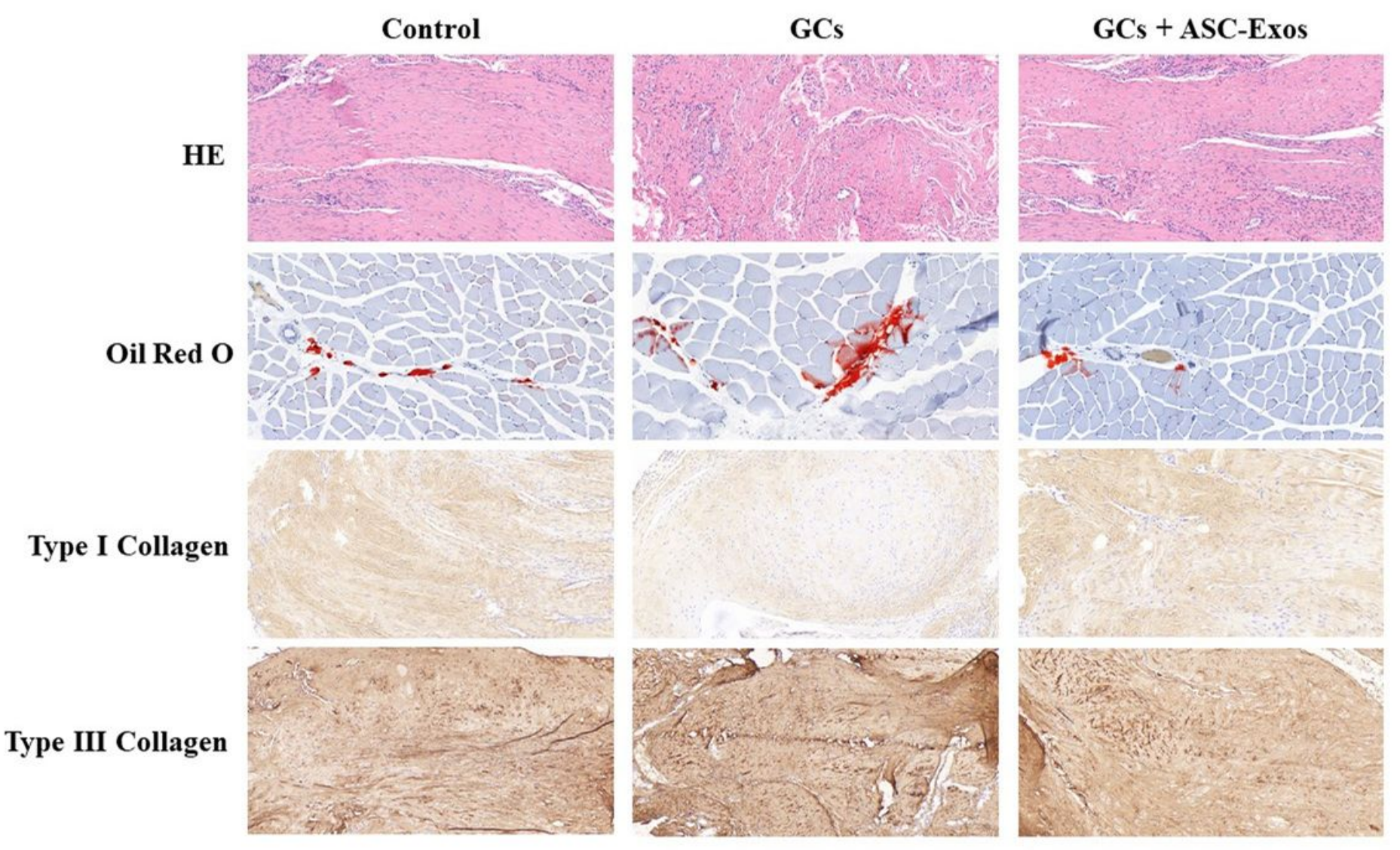

Figure 8

Histological analysis including (A) HE staining, (B) Oil Red O staining, (C) Type I collagen staining, and (D) Type III collagen staining of injured supraspinatus in the saline treated group (Control), GCs treated group (GCs), and GCs 


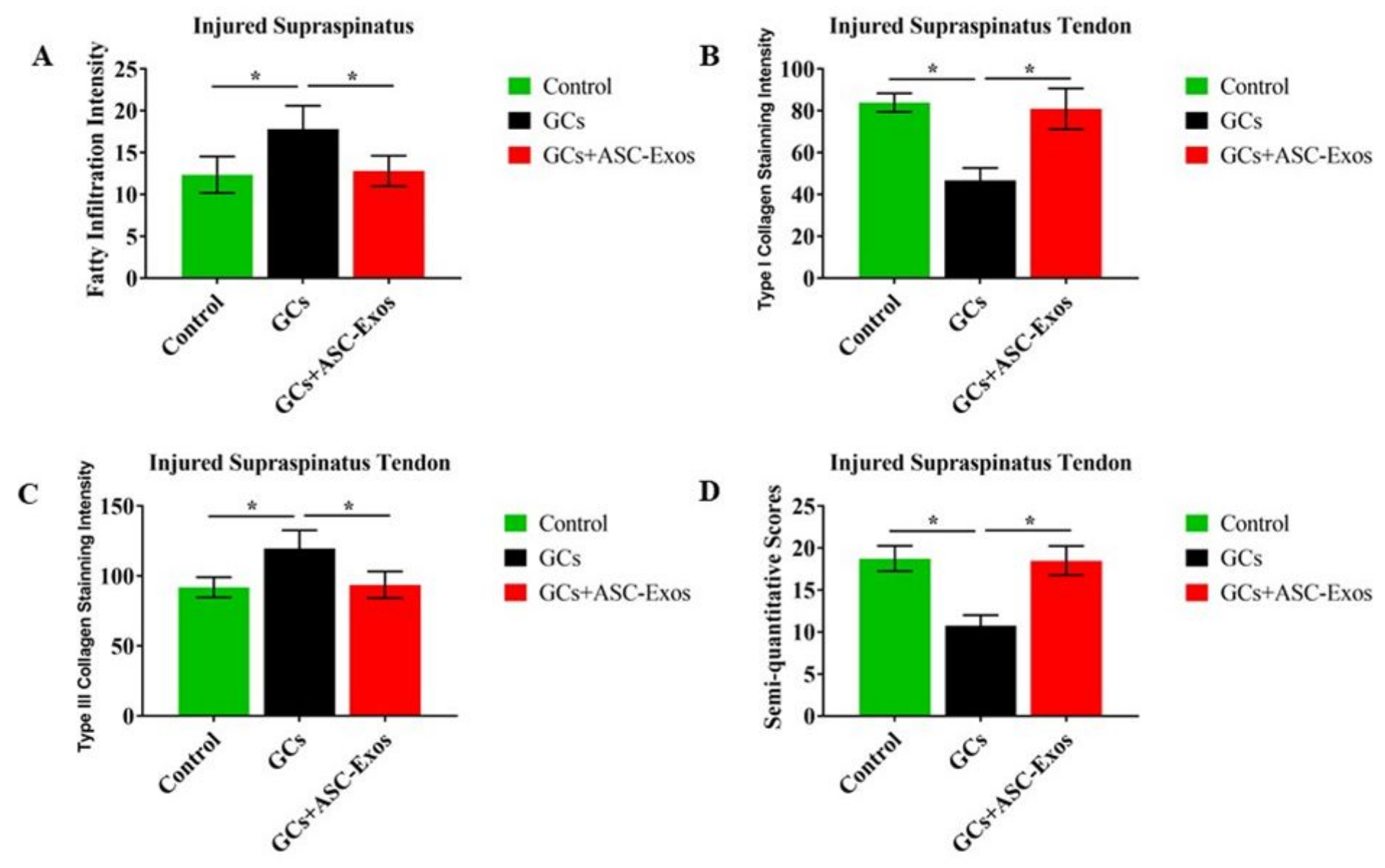

Figure 9

Quantitative analysis of (A) fatty infiltration intensity, (B) type I collagen staining intensity, (C) type III collagen staining intensity, and (D) semi-quantitative analysis of injured supraspinatus in the saline treated group (Control), GCs treated group (GCs), and GCs + ASC-Exos treated group (GCs + ASC-Exos). Data are expressed as mean $\pm S D$. * indicates significant differences, $P<0.05$. 


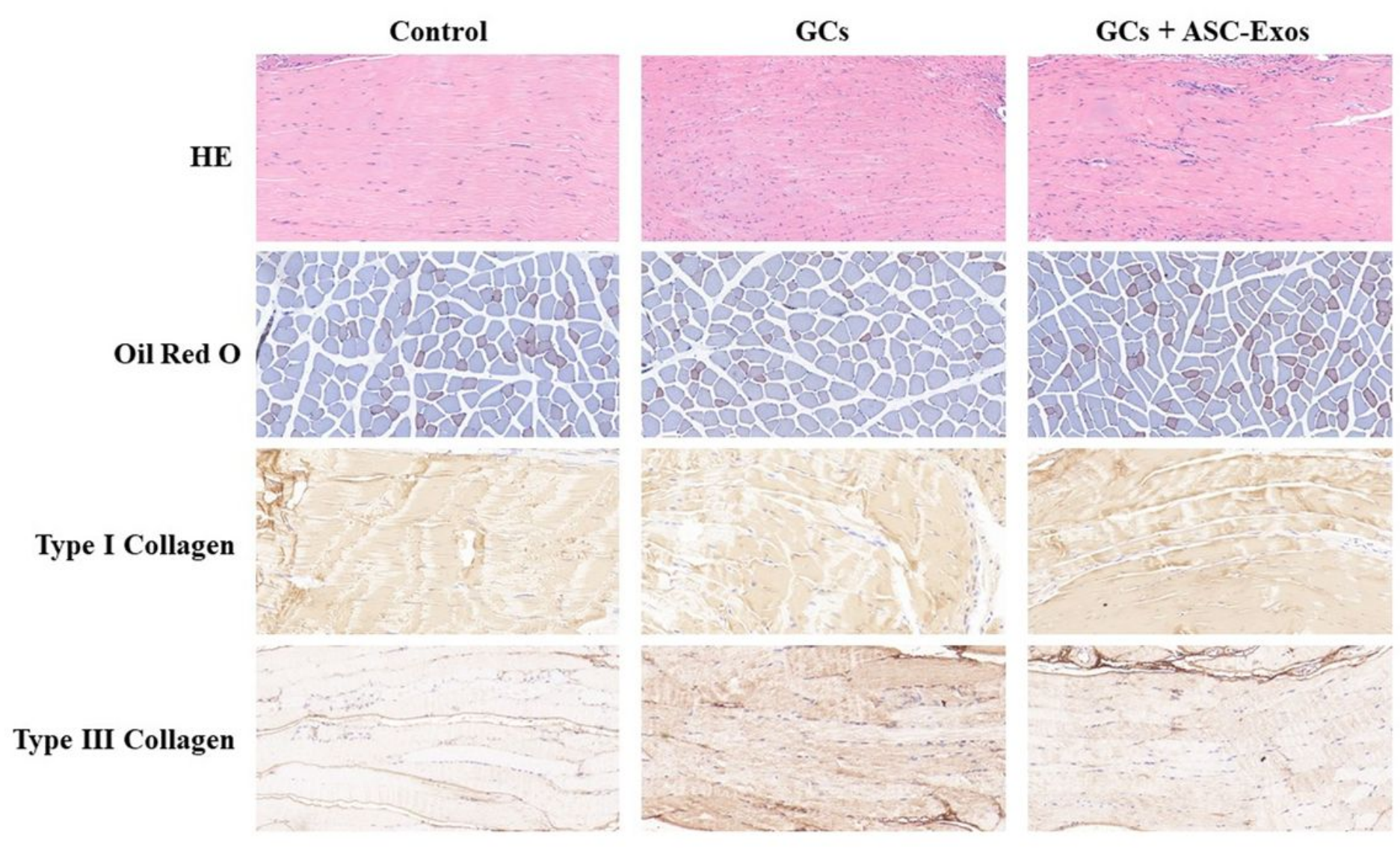

Figure 10

Histological analysis including (A) HE staining, (B) Oil Red O staining, (C) Type I collagen staining, and (D) Type III collagen staining of intact infraspinatus in the saline treated group (Control), GCs treated group (GCs), and GCs + ASC-Exos treated group (GCs + ASC-Exos). Magnification 4x 

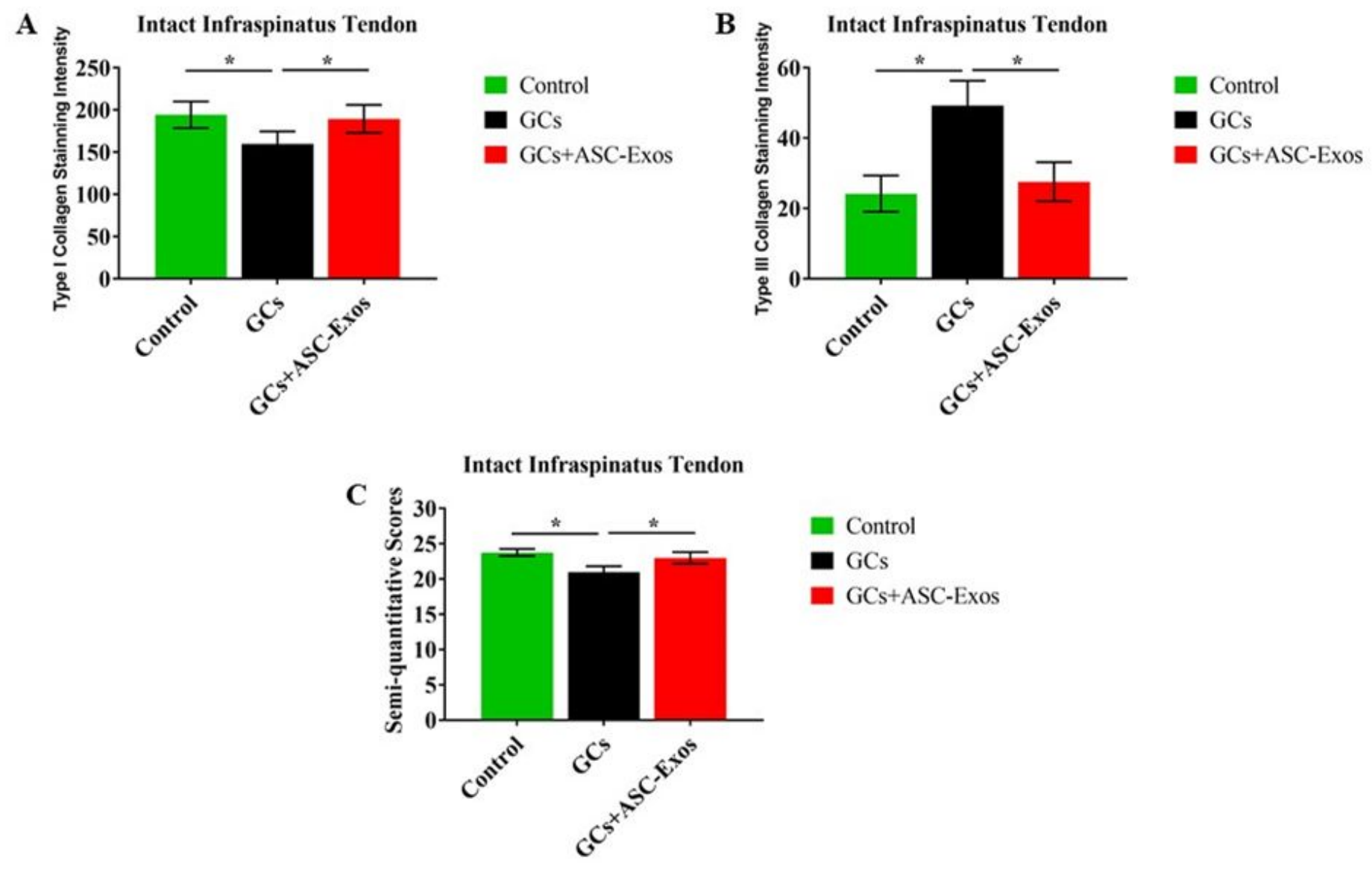

\section{Figure 11}

Quantitative analysis of (A) type I collagen staining intensity, (B) type III collagen staining intensity, and (C) semi-quantitative analysis of intact infraspinatus tendon in the saline treated group (Control), GCs treated group (GCs), and GCs + ASC-Exos treated group (GCs + ASC-Exos). Data are expressed as mean \pm SD. * indicates significant differences, $P<0.05$.
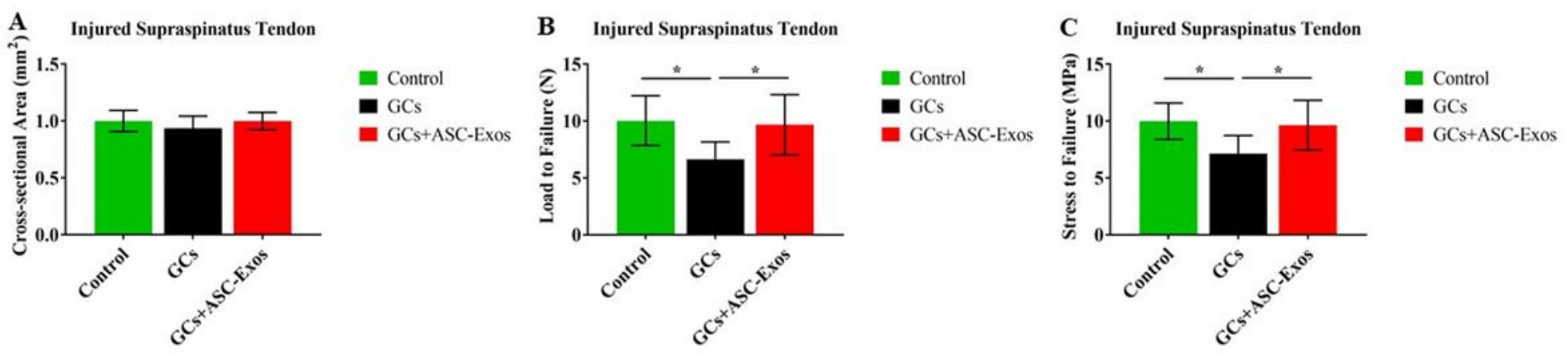

Figure 12 
Biomechanical analysis of (A) cross-sectional area, (B) load-to-failure, and (C) stress-to-failure of injured supraspinatus tendon in the saline treated group (Control), GCs treated group (GCs), and GCs + ASC-Exos treated group (GCs + ASC-Exos). Data are expressed as mean $\pm \mathrm{SD}$. * indicates significant differences, $\mathrm{P}<0.05$.
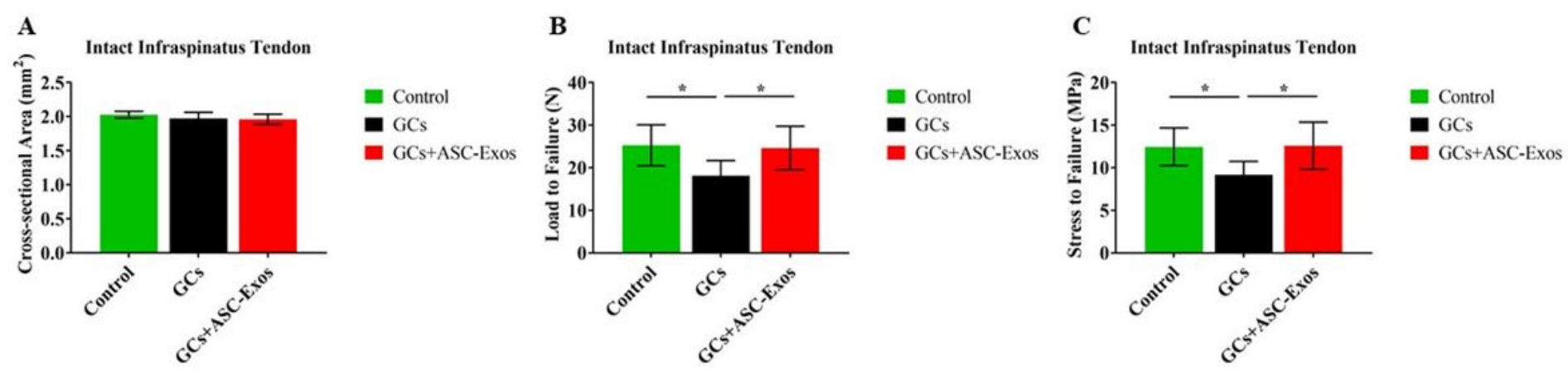

Figure 13

Biomechanical analysis of (A) cross-sectional area, (B) load-to-failure, and (C) stress-to-failure of intact infraspinatus tendon in the saline treated group (Control), GCs treated group (GCs), and GCs + ASC-Exos treated group (GCs + ASC-Exos). Data are expressed as mean \pm SD. * indicates significant differences, $\mathrm{P}<0.05$. 